\title{
Methionine Sulfoxide Reduction and the Aging Process
}

\author{
AHMET KOC $^{a}$ AND VADIM N. GLADYSHEV ${ }^{b}$ \\ ${ }^{a}$ Department of Biology, Izmir Institute of Technology, 35430 Urla, Izmir, Turkey \\ ${ }^{b}$ Department of Biochemistry, University of Nebraska, Lincoln, Nebraska 68588, \\ USA
}

\begin{abstract}
Aging has been described for multicellular and asymmetrically dividing organisms, but the mechanisms are poorly understood. Oxidation of proteins is considered to be one of the major factors that leads to aging. Oxidative damage to proteins results in the oxidation of certain amino acid residues, among which oxidation of sulfur-containing amino acids, methionine and cysteine, is notable because of the susceptibility of these residues to damage, and occurrence of repair mechanisms. Methionine sulfoxide reductases, MsrA and MsrB, are thioredoxin-dependent oxidoreductases that reduce oxidized forms of methionine, methionine sulfoxides, in a stereospecific manner. These enzymes are present in all cell types and have shown to be regulating life spans in mammals, insects, and yeast. Here, their roles in modulating yeast life span are discussed.
\end{abstract}

KEYWORDS: aging; ROS; methionine sulfoxide reductase; anaerobic; yeast life span

\section{INTRODUCTION}

Methionine residues in proteins are easily oxidized by reactive oxygen species (ROS) to methionine-S-sulfoxides and methionine-R-sulfoxides, and these modified residues are reduced back to methionine by antioxidant enzymes, methionine-S-sulfoxide reductase (MsrA) and methionine-R-sulfoxide reductase (MsrB), respectively. ${ }^{1,2}$ Recent studies revealed that methionine sulfoxide reduction provides life-span extension in animals; deletion of the MSRA gene in mice reduced life span by $40 \%,{ }^{3}$ whereas MsrA overexpression in the central nervous system in Drosophila melanogaster extended life span by $\sim 70 \%$. $^{4}$ In addition to life-span modulation, comparison of samples taken from young and old animals revealed that there is an age-related decrease in Msr $\operatorname{level}^{5,6}$ and an increase in the amount of methionine sulfoxides in different

Address for correspondence: Ahmet Koc, Department of Biology, Izmir Institute of Technology, 35430 Urla, Izmir, Turkey. Voice: 90-232-750-7544; fax: 90-232-750-7509.

ahmetkoc@iyte.edu.tr

Ann. N.Y. Acad. Sci. 1100: 383-386 (2007). (C) 2007 New York Academy of Sciences.

doi: 10.1196/annals.1395.042 
tissues. ${ }^{7,8}$ These studies demonstrate the importance of methionine sulfoxide reduction in the aging process.

\section{YEAST METHIONINE SULFOXIDE REDUCTASES}

The budding yeast $S$. cerevisiae has been extensively used as a model organism in aging studies. ${ }^{9,10}$ Only a single MSRA (Mxr1, YER042W) and $M S R B$ (YCL033C) genes occurs in yeast. MsrA mutant cells cannot efficiently reduce protein-based methionine sulfoxides and only partially reduce free methionine sulfoxides. ${ }^{11}$ MsrB mutant cells have more mild phenotypes, however, double mutants lacking both MSRA and MSRB genes cannot grow on methionine-S-sulfoxide as the sole source of sulfur, suggesting that MsrA is the only methionine-S-sulfoxide-specific reductase. However, there is a residual growth of double mutants in the presence of methionineR-sulfoxide suggesting the presence of an additional minor MsrB activity. Identity of the protein that catalyzes this reaction is not known. ${ }^{12}$ Recombinant yeast MsrA protein is 10 -fold more active than recombinant MsrB in in vitro reduction of dabsylated L-methionine sulfoxide to dabsylated methionine. Consistent with its high specific activity, deletion of MsrA results in stronger hydrogen peroxide sensitivity than deletion of MsrB. ${ }^{12}$

\section{ROLE OF MSRA AND MSRB IN YEAST AGING}

Although deletion of MsrA decreases and overexpression increases the life span by $25 \%$, deletion or overexpression of MsrB has no effects under normal growth conditions. However, simultaneous deletion of both genes dramatically reduces the life span by $63 \%$ suggesting a role of $\mathrm{MsrB}$ in the absence of MsrA. Remarkably, overexpression of MsrB under calorie-restriction conditions extends life span by $120 \%{ }^{12}$ Primary structure of MsrB contains a signal peptide that is suggestive of mitochondrial location. Since calorie restriction extends the life span by increasing the mitochondrial respiration rate, MsrB may be crucial for regulating this process. It should be noted that $\mathrm{MsrB}$ is a selenium-containing protein in mammals ${ }^{2}$ and its expression is regulated by dietary levels of this trace element. Thus, the dramatic lifespan extension in the yeast offered by MsrB under calorie-restriction conditions suggests that dietary selenium supplements alone or in combination with restricted food intake are good candidates for dietary life extension in mammals.

To further analyze the role of MsrA and MsrB in aging, life-span assays were carried out under anaerobic conditions, where ROS production is low. As opposed to the expected extension of life span, cells lived 35\% shorter 
under anaerobic conditions and neither deletion nor overexpression of MsrA or MsrB had modulatory effects on the life span. A possible explanation for shorter life span under anaerobic condition is that the cells may not derive sufficient energy to drive biochemical reactions, because oxidative phosphorylation requires oxygen for ATP synthesis and anaerobic yeast can only use glycolysis to produce a limited amount of energy. Further reduction $(15 \%)$ of life span by calorie restriction under anaerobic condition clearly shows the link between the ability for efficient energy production and life span. ${ }^{12}$

\section{CONCLUDING REMARKS}

Methionine sulfoxide reductases are antioxidant enzymes that play a role in scavenging ROS damage by repairing oxidized methionine residues. Their absence increases the level of ROS damage and decreases the mean and maximum life span. This work suggested that ROS should be considered as modulators rather than causes of aging. Even though aerobic metabolism is accompanied by ROS production, it results in a longer life span, most likely because of efficient energy production.

\section{REFERENCES}

1. Weissbach, H. et al. 2002. Peptide methionine sulfoxide reductase: structure, mechanism of action, and biological function. Arch. Biochem. Biophys. 397: $172-178$.

2. KRYUKOV, G.V. et al. 2002. Selenoprotein R is a zinc-containing stereo-specific methionine sulfoxide reductase. Proc. Natl. Acad. Sci. USA 99: 4245-4250.

3. Moskovitz, J. et al. 2001. Methionine sulfoxide reductase (MsrA) is a regulator of antioxidant defense and lifespan in mammals. Proc. Natl. Acad. Sci. USA 98: 12920-12925.

4. RuAN, H. et al. 2002. High-quality life extension by the enzyme peptide methionine sulfoxide reductase. Proc. Natl. Acad. Sci. USA 99: 2748-2753.

5. StadtMan, E.R. et al. 2002. Cyclic oxidation and reduction of protein methionine residues is an important antioxidant mechanism. Mol. Cell Biochem. 234-235: 3-9.

6. Petropoulos, I. et al. 2001. Rat peptide methionine sulphoxide reductase: cloning of the cDNA, and down-regulation of gene expression and enzyme activity during aging. Biochem. J. 355: 819-825.

7. GARNER, M.H. \& A. SPECTOR. 1980. Selective oxidation of cysteine and methionine in normal and senile cataractous lenses. Proc. Natl. Acad. Sci. USA 77: 12741277.

8. Horstmann, H.J., J.W. Rohen \& K. Sames. 1983. Age-related changes in the composition of proteins in the trabecular meshwork of the human eye. Mech. Ageing Dev. 21: 121-136. 
9. Tissenbaum, H.A. \& L. Guarente. 2002. Model organisms as a guide to mammalian aging. Dev. Cell 2: 9-19.

10. JAZWINSKI, S.M. 2002. Growing old: metabolic control and yeast aging. Annu. Rev. Microbiol. 56: 769-792.

11. Moskovitz, J. et al. 1997. The yeast peptide-methionine sulfoxide reductase functions as an antioxidant in vivo. Proc. Natl. Acad. Sci. USA 94: 9585-9589.

12. Koc, A. et al. 2004. Methionine sulfoxide reductase regulation of yeast lifespan reveals reactive oxygen species-dependent and -independent components of aging. Proc. Natl. Acad. Sci. USA 101: 7999-8004. 